\title{
PROJECTIVE TOPOLOGICAL GROUPS ${ }^{1}$
}

\author{
C. E. HALL
}

The notion of a projective topological group for a category of topological groups has been defined by Hofmann [4]. Free topological groups have been of interest in the study of topological groups since their invention by Markov [5]. It is the purpose of this paper to develop the concept of projective in the category of Abelian topological groups, based upon the requirement that the class of projective topological groups contains the class of free Abelian topological groups. Thus the approach used here is necessarily different from that taken by Hofmann [4]. We also will show that the class of projectives so obtained properly contains the class of free Abelian topological groups, and will give an example to show that the direct sum of projective topological groups may fail to be projective.

Notations. All topological groups considered are Abelian and Hausdorff. Any unexplained notation or terminology is that of Hewitt and Ross [3]. If $X$ is a completely regular space then $A(X)$ denotes the free Abelian topological group on $X$ as defined by Markov [5] and $A_{G}(X)$ denotes the free Abelian topological group on $X$ as defined by Graev [2]. The words "free topological group" mean a free topological group as defined by Markov [5]. If $B$ is a topological group, $\alpha_{B}$ denotes the open continuous homomorphism from $A(B)$ onto $B$ defined by $\alpha_{B}(b)=b$ all $b \in B$. If $I$ is an index set and $P_{i}$ is a topological group for each $i \in I$ then by $\sum P_{i}(i \in I)$ we mean the direct sum of $\left\{P_{i}: i \in I\right\}$ with the relativized product topology. If $I=\{1,2, \cdots, n\}$, then $\sum P_{i}(i \in I)$ is written as $P_{1} \times P_{2} \times \cdots \times P_{n}$. A sequence of the form $f: A \rightarrow B \rightarrow 0$ is exact if $A$ and $B$ are topological groups and $f$ is a continuous homomorphism from $A$ on to $B$.

DEFINITION 1. A topological group $G$ is projective relative to a family $F$ of exact sequences of the form

$$
A \stackrel{f}{\rightarrow} B \rightarrow 0
$$

iff for each (1) $\in F$ and each continuous homomorphism $g$ from $G$ to $B$ there is a continuous homomorphism $h$ from $G$ to $A$ such that $f h=g$. Let $\varepsilon$ be the family of all exact sequences of the form (1) such that all free Abelian topological groups are projective relative to $\varepsilon$. A topo-

Presented to the Society, January 25, 1966; received by the editors June 7, 1966.

1 This paper is a revision of part of the author's Doctoral dissertation written at New Mexico State University under the direction of E. D. Gaughan. 
logical group is projective iff it is projective relative to $\varepsilon$.

Definition 2. Let $G$ be a topological group. A topological group $H$ is said to be a summand of $G$ iff there is a topological group $K$ such that $G \cong H \times K$.

The following two theorems together with the examples given later contain the main results of this paper.

THEOREM 1. A topological group $P$ is projective iff it is a summand of a free Abelian topological group.

THEOREM 2. Let $P$ be a projective topological group and suppose that $P$ is algebraically the free Abelian group on $X=\left\{x_{1} \cdots, x_{n}\right\}$. Then $P$ is the free Abelian topological group on $X$.

LEMmA 1. (1) is in $\mathcal{E}$ iff there is a continuous function $g: B \rightarrow A$ such that $f g=1_{B}$.

Proof. If (1) is in $\varepsilon$ there is a continuous homomorphism $h$ from $A(B)$ to $A$ such that $f h=\alpha_{B}$. Then $g=h \mid B$ is a continuous function from $B$ to $A$ and $f g=1_{B}$.

Suppose that $g$ is a continuous function from $B$ to $A$ such that $f g=1_{B}$. Let $X$ be a completely regular space and $h$ be a continuous homomorphism from $A(X)$ to $B$. Let $\beta=g(h \mid X)$. Then $\beta$ is a continuous function from $X$ to $A$ and so, by definition of $A(X)$, may be extended to a continuous homomorphism $\beta^{\prime}$ from $A(X)$ to $A$. For $x \in X$, $\left(f \beta^{\prime}\right)(x)=f(\beta(x))=(f g)(h(x))=h(x)$ so that $h$ and $f \beta^{\prime}$ are homomorphisms from $A(X)$ to $B$ which agree on $X$. Thus $h=f \beta^{\prime}$ and we have that (1) is in $\varepsilon$.

Note that the function $g$ in Lemma 1 is not necessarily a homomorphism. It follows from this lemma that

$$
\left(A(B) \stackrel{\alpha_{B}}{\longrightarrow} B \rightarrow 0\right) \in \mathcal{E}
$$

for every topological group $B$.

Lemma 2. Let $\left\{P_{i}: i \in I\right\}$ be a family of topological groups and $P=\sum P_{i}(i \in I)$. If $P$ is projective then each $P_{i}$ is projective.

Proof. Suppose (1) is in $\varepsilon$. Let $i \in I$ and denote by $\phi_{i}$ the projection of $P$ onto $P_{i}$. Suppose $g: P_{i} \rightarrow B$ is a continuous homomorphism. Since $P$ is projective there is a continuous homomorphism $h: P \rightarrow A$ such that $f h=g \phi_{i}$. Let $k=h \mid P_{i}$. Then $k$ is a continuous homomorphism from $P_{i}$ into $A$ and $f k=g$.

Lemma 3. Let $P$ and $G$ be topological groups and suppose $f$ is a continuous homomorphism of $G$ onto $P$. If there is a continuous homomorphism 
$g: P \rightarrow G$ such that $f g=1_{P}$ then $G \cong P \times \operatorname{Ker} f$.

Proof. The function $g$ is one-to-one and onto $g[P]$. Then the continuous function $g f$ maps $G$ onto $g[P]$ and if $g(p) \in g[P]$ then $(g f)(g(p))=g(p)$. Thus $g[P]$ is a closed subgroup and it follows from this $\left[3\right.$, p. 59] that $G \cong g[P] \times(g f)^{-1}(\{0\})$. Now $(g f)^{-1}(\{0\})=\operatorname{Ker} f$. Also $g^{-1}=f \mid g[P]$ hence $P$ is topologically isomorphic to $g[P]$. Thus $G \cong P \times \operatorname{Ker} f$.

Proof of Theorem 1. That summands of free Abelian topological groups are projective follows immediately from Lemma 2.

Suppose $P$ is projective. Since

$$
\left(A(P) \stackrel{\alpha_{P}}{\longrightarrow} P \rightarrow 0\right) \in \mathcal{E}
$$

there is a continuous homomorphism $f: P \rightarrow A(P)$ such that $\alpha_{P} f=1_{P}$. Then by Lemma $3, A(P) \cong P \times \operatorname{Ker} \alpha_{P}$.

Corollary 1. A projective topological group is algebraically a free Abelian group.

Corollary 2. A projective topological group which is not discrete is neither a locally compact nor a complete metric space.

Corollary 2 is an immediate consequence of Corollary 1 and results of Dudley [1].

Proof of Theorem 2. Since $P$ is projective there is a continuous homomorphism $f: P \rightarrow A(P)$ such that $\alpha_{P} f=1_{P}$. Now $f\left(x_{j}\right)$ $=\sum_{k=1}^{r(j)} n(j, k) m_{j, k}$ where $n(j, k) \in Z ; m_{j, k} \in P, k=1, \cdots, r(j)$; for $j=1, \cdots, n$. Also note that $m_{j, k}=\sum_{q=1}^{n} s(j, k, q) x_{q}$ where $s(j, k, q)$ $\in Z$ for $j, q \in\{1, \cdots, n\}, k=1, \cdots, r(j)$.

Suppose $B$ is a topological group and $g: X \rightarrow B$ is a continuous function. Let $g\left(x_{q}\right)=b_{q}$ for $1 \leqq q \leqq n$. We shall show that $g$ can be extended to a continuous homomorphism $g^{\prime}: P \rightarrow B$, and hence that $P$ is the free Abelian topological group on $X$.

Since $P$ is a countably infinite completely regular space there are open and closed sets $\left\{U_{j, k}\right\}, 1 \leqq j \leqq n, 1 \leqq k \leqq r(j)$, such that $m_{j, k}$ $\in U_{j, k}$. The $U_{j, k}$ may also be chosen such that $U_{j, k} \cap U_{u, v}=\varnothing$ if $m_{j, k} \neq m_{u, v}$ and $U_{j, k}=U_{u, v}$ if $m_{j, k}=m_{u, v}$.

Then the function $h: P \rightarrow B$ defined by $h(y)=\sum_{q=1}^{n} s(j, k, q) b_{q}$ if $y \in U_{j, k}$ for some $1 \leqq j \leqq n, 1 \leqq k \leqq r(j)$ and $h(y)=0$ if $y \in\left(P \backslash \bigcup_{j, k} U_{j, k}\right)$ is continuous. Thus $h$ has an extension $h^{\prime}$ to a continuous homomorphism from $A(P)$ into $B$.

Let $g^{\prime}=h^{\prime} f$. Then $g^{\prime}$ is a continuous homomorphism from $P$ in to $B$. It remains to show that $g^{\prime} \mid X=g$. Since $P$ is algebraically free on $X$ 
there is a group homomorphism $\theta: P \rightarrow B$ such that $\theta \mid X=g$. Suppose $x_{j} \in X$. Then

$$
\begin{aligned}
g^{\prime}\left(x_{j}\right) & =h^{\prime}\left(f\left(x_{j}\right)\right)=h^{\prime}\left(\sum_{k=1}^{r(j)} n(j, k) m_{j, k}\right)=\sum_{k=1}^{r(j)} n(j, k) h^{\prime}\left(m_{j, k}\right) \\
& =\sum_{k=1}^{r(j)} n(j, k)\left[\sum_{q=1}^{n} s(j, k, q) b_{q}\right]=\theta\left(\sum_{k=1}^{r(j)} n(j, k) m_{j, k}\right) \\
& =\theta\left(\left(\alpha_{P} f\right)\left(x_{j}\right)\right)=\theta\left(x_{j}\right)=b_{j}=g\left(x_{j}\right) .
\end{aligned}
$$

An example of a projective topological group that is not a free Abelian topological group will now be constructed.

EXAMPLE 1. Let $X$ be a completely regular Hausdorff space with the following properties:

(1) $X=X_{1} \cup X_{2}$ where $X_{1}$ and $X_{2}$ are connected completely regular spaces containing more than one element;

(2) $X_{1} \cap X_{2}=\varnothing$;

(3) $X_{1}$ and $X_{2}$ are both open and closed in $X$.

Form the group $A_{G}(X)$ letting $0^{\prime}$, the zero element of the group $A_{G}(X)$, be an element of $X_{1}$. Define a function $f: X \rightarrow Z$ by $f(x)=0$ if $x \in X_{1}$ and $f(x)=1$ if $x \in X_{2}$. Then $f$ is continuous and $f\left(0^{\prime}\right)=0$; hence $f$ may be extended to a continuous homomorphism $f^{\prime}$ mapping $A_{G}(X)$ onto $Z$. Then it is an immediate consequence of Lemma 3 that $A_{G}(X)$ $\cong Z \times \operatorname{Ker} f^{\prime}$.

Since $Z$ is discrete and $f^{\prime}$ is continuous, $\operatorname{Ker} f^{\prime}$ is an open and closed subgroup of $A_{G}(X)$. Let $C$ denote the component of $0^{\prime}$ in $A_{G}(X)$. We will verify that $C=\operatorname{Ker} f^{\prime}$. Since $\operatorname{Ker} f^{\prime}$ is an open subgroup of $A_{G}(X)$ it is immediate that $C \subseteq \operatorname{Ker} f^{\prime}$. Observe that

$$
\begin{aligned}
\operatorname{Ker} f^{\prime}=\left\{\left(\sum_{i=1}^{n} n_{i} x_{i}+\sum_{i=1}^{m} m_{i} y_{i}\right)\right. & \in A_{G}(X): \\
& \left.x_{i} \in X_{1} \backslash\{0\}, y_{i} \in X_{2}, \sum_{i=1}^{m} m_{i}=0\right\} .
\end{aligned}
$$

Let $x \in X_{2}$. Then the set $\left(X_{2}-x\right)=\left\{(y-x): y \in X_{2}\right\}$ algebraically generates

$$
\left\{\sum_{i=1}^{m} m_{i} y_{i} \in A_{G}(X): y_{i} \in X_{2}, \sum_{i=1}^{m} m_{i}=0\right\}
$$

and therefore the set $X_{1} \cup\left(X_{2}-x\right)$ algebraically generates $\operatorname{Ker} f^{\prime}$. Now $X_{1}$ is connected and $0^{\prime} \in X_{1}$, hence $X_{1} \subseteq C$. Also $\left(X_{2}-x\right)$ is connected and $0^{\prime} \in\left(X_{2}-x\right)$, hence $\left(X_{2}-x\right) \subseteq C$. Since $C$ is a subgroup of $A_{G}(X)$ it follows that $\operatorname{Ker} f^{\prime} \subseteq C$. 
According to a result of Graev $[2, \S 5]$, since $X$ is not connected, there is a completely regular space $X^{\prime}$ such that $A\left(X^{\prime}\right) \cong A_{G}(X)$. So $A\left(X^{\prime}\right) \cong Z \times C$ and thus $C$ is projective. But $C$ is also connected and hence $C$ cannot be a free Abelian topological group [5, Theorem 26].

It is easy to see that the converse of Lemma 2 is true if the set $I$ is finite. It should be noted, however, that it is not valid in general.

EXAmPLE 2. Let $X_{n}=\left[x_{n}\right]$ be an infinite cyclic group with the discrete topology for $n=1,2, \cdots$. Of course, each $X_{n}$ is projective. Let $P=\sum_{n=1}^{\infty} X_{n}$. We will show that $P$ is not projective.

Assume that $P$ is projective; then there is a continuous homomorphism $g$ from $P$ into $A(P)$ such that $\alpha_{P} g=1_{P}$. For each $n$, let $k_{n}$ $=x_{n}-g\left(x_{n}\right)$. Then, by the continuity of $g$ and the definition of the product topology on $P,\left\{k_{n}\right\}_{n=1}^{\infty}$ is a sequence in $\operatorname{Ker} \alpha_{P}$ such that if $U$ is a neighborhood of 0 in $A(P)$ then $\left\{n: Y_{n} \cap U \neq Y_{n}\right\}$ is finite where $Y_{n}=\left[x_{n}-k_{n}\right] \subseteq A(P)$. Let $0^{\prime}$ denote the identity element of $P$ and $X=\left\{x_{1}, x_{2}, \cdots\right\}$. Then $X \cup\left\{0^{\prime}\right\}$ is closed in $P$, hence closed in $A(P)$ and $0_{A(P)} \notin X \cup\left\{0^{\prime}\right\}$. Thus $\left\{n: k_{n}=0\right\}$ is finite. Since $k_{j} \in A(P), k_{j}$ can be written uniquely in the form

$$
k_{j}=\sum_{i=1}^{N(j)} m_{j i} p_{j i} ; p_{j i} \in P \quad \text { for } 1 \leqq i \leqq N(j) .
$$

Note that there is an integer $n_{0}$ such that for $n \geqq n_{0}, x_{n}$ $\notin\left\{p_{11}, p_{12}, \cdots, p_{1 N(1)}\right\}$.

Suppose that there does not exist $n \geqq n_{0}$ such that $x_{n} \neq m_{n i} p_{n i}$ for all $1 \leqq i \leqq N(n)$. If this happens we claim there is $n \geqq n_{0}$ such that $p_{n i}$ $\notin\left\{p_{11}, p_{12}, \cdots, p_{1 N(1)}, x_{n}\right\}$ for some $1 \leqq i \leqq N(n)$. Suppose not. Then for every $n \geqq n_{0},\left(x_{n}-k_{n}\right)=x_{n}-\sum_{i=1}^{N(n)} m_{n i} p_{n i}$ (and without loss of generality) $\left(x_{n}-k_{n}\right)=\sum_{i=2}^{N(n)} m_{n i} p_{n i}, x_{n} \in\left\{p_{n 2}, \cdots, p_{n N(n)}\right\}$ and $\left\{p_{n 2}, \cdots, p_{n N(n)}\right\} \subseteq\left\{p_{11}, p_{12}, \cdots, p_{1 N(1)}\right\}$. Thus $x_{n}=\alpha_{P}\left(x_{n}-k_{n}\right)$ $=\sum_{i=2}^{N(n)} m_{n i} p_{n i} \in P$ for every $n \geqq n_{0}$. Now there is a finite set $\{j(1), \cdots, j(m)\}$ of natural numbers such that the subgroup $H$ $=\left[\left\{p_{11}, \cdots, p_{1 N(1)}\right\}\right]$ of $P$ is contained in the subgroup $T$ $=\left[\left\{x_{j(1)}, \cdots, x_{j(m)}\right\}\right]$ of $P$. Then for every $n \geqq n_{0}, x_{n} \in H$, but also there is $n \geqq n_{0}$ such that $x_{n} \notin T$. This is a contradiction.

We define inductively a subsequence $\left\{y_{n}\right\}_{n=1}^{\infty}$ of $\left\{x_{n}-k_{n}\right\}_{n=1}^{\infty}$ in the following manner: Let $y_{1}=x_{1}-k_{1}$. Suppose $y_{1}, y_{2}, \cdots, y_{n}$ have been selected, $y_{i}=x_{q(i)}-k_{q(i)}$ for $1 \leqq i \leqq n$. Let $y_{n+1}=x_{t}-k_{t}$ where $t$ is the least integer such that $t>q(i)$ for $1 \leqq i \leqq n$, and such that

$$
x_{t} \notin S=U\left\{\left\{p_{j 1}, \cdots, p_{j N(j)}\right\}: j \in\{1, q(2), \cdots, q(n)\}\right\}
$$

and $x_{t} \neq m_{t i} p_{t i}$ for $1 \leqq i \leqq N(t)$; if such exists. If this fails, there is a least $t>q(i), 1 \leqq i \leqq n$, such that $x_{t} \notin S$ and for some $i, 1 \leqq i \leqq N(t)$, 
$p_{t i} \notin S \cup\left\{x_{t}\right\}$. We use this choice of $t$ if the former does not exist.

Relabel so that $y_{n}=t_{n}-r_{n}, t_{n} \in X, r_{n}=\sum_{i=1}^{m(n)} m_{n i} p_{n i}, p_{n i} \in P$. Define $U_{n}=2^{n}\left(t_{n}-r_{n}\right)$.

By induction we define a sequence of real-valued continuous functions $\left\{f_{i}\right\}_{i=1}^{\infty}$ on $P$ with the following properties: Let $f_{11}(x)=\frac{1}{2}$ for all $x \in P$. For $i>1$ we have:

A. The absolute value of the function $f_{i}$ does not exceed $2^{-i}$;

B. $f_{i}\left(t_{j}\right)=0$ for $1 \leqq j \leqq(i-1)$;

C. $f_{i}\left(\cup_{\boldsymbol{k}=1}^{m(i-1)}\left\{p_{k 1}, \cdots, p_{k m(k)}\right\}\right)=0$.

D. (i) If $t_{i} \notin T=\left\{p_{i 1}, \cdots, p_{i m(i)}\right\}$ then $f_{i}(T)=0$ and $f_{i}\left(t_{i}\right)= \pm 2^{-i}$ where the sign is to be determined in such a way that it agrees with the sign of

$$
\sum_{j=1}^{i-1}\left[f_{j}\left(t_{i}\right)-\sum_{s=1}^{m(i)} m_{i s} f_{j}\left(p_{i s}\right)\right]
$$

(ii) If $t_{i}=p_{i j}$ for some $1 \leqq j \leqq m(i)$ and $m_{i j} \neq 1$, then $f_{i}\left(T \backslash\left\{p_{i j}\right\}\right)=0$ and $f_{i}\left(t_{i}\right)= \pm 2^{-i}$ where the sign is to be determined in such a way that the sign of $\left(1-m_{i j}\right) f_{i}\left(t_{i}\right)$ agrees with the sign of $(*)$.

(iii) If $t_{i}=p_{i k}$ for some $1 \leqq k \leqq m(i)$ and $m_{i k}=1$, there is $p_{i j} \in T$ such that $\left.p_{i j} \notin\left[\bigcup_{k=1}^{m(i-1)}\left\{p_{k 1}, \cdots, p_{k m(k)}\right\}\right) \cup\left\{t_{i}\right\}\right]$. Then $f_{i}\left(T \backslash\left\{p_{i j}\right\}\right)=0$, $f_{i}\left(t_{i}\right)=0$, and $f_{i}\left(p_{i j}\right)= \pm 2^{-i}$ where the sign is to be determined in such a way that it agrees with the sign of $(*)$.

We consider the real-valued function $f$ on $P$ defined by $f(x)$ $=\sum_{n=1}^{\infty} f_{n}(x), x \in P$. The function $f$ is con tinuous on $P$. Let $f^{\prime}$ denote the extension of $f$ to a continuous homomorphism mapping $A(P)$ into $R$ (additive group of real numbers). We now examine $f^{\prime}\left(U_{n}\right)$ $=2^{n} f^{\prime}\left(t_{n}-r_{n}\right)$ for $n \geqq 2$. There are three cases according as $t_{n}$ satisfies $\mathrm{D}(\mathrm{i})$, $\mathrm{D}(\mathrm{ii})$, or $\mathrm{D}(\mathrm{iii})$.

$$
\begin{aligned}
\left|f^{\prime}\left(U_{n}\right)\right| & =2^{n}\left|\sum_{j=1}^{\infty}\left[f_{j}^{\prime}\left(t_{n}\right)-f_{j}^{\prime}\left(r_{n}\right)\right]\right| \\
& =2^{n}\left|\sum_{j=1}^{n-1}\left[f_{j}^{\prime}\left(t_{n}\right)-f_{j}^{\prime}\left(r_{n}\right)\right]+f_{n}{ }^{\prime}\left(t_{n}\right)\right| \\
& \geqq 2^{n}\left|f_{n}\left(t_{n}\right)\right|=1 . \\
\left|f^{\prime}\left(U_{n}\right)\right| & =2^{n}\left|\sum_{j=1}^{n-1}\left[f_{j}^{\prime}\left(t_{n}\right)-f_{j}^{\prime}\left(r_{n}\right)\right]+\left(1-m_{n j}\right) f_{n}\left(t_{n}\right)\right| \\
& \geqq 2^{n}\left|\left(1-m_{n j}\right)\right| \cdot\left|f_{n}\left(t_{n}\right)\right| \geqq 1 . \\
\left|f^{\prime}\left(U_{n}\right)\right| & =2^{n}\left|\sum_{j=1}^{n-1}\left[f_{j}^{\prime}\left(t_{n}\right)-f_{j}^{\prime}\left(r_{n}\right)\right]+f_{n}\left(p_{n j}\right)\right| \\
& \geqq 2^{n}\left|f_{n}\left(p_{n j}\right)\right|=1 .
\end{aligned}
$$


Thus the identity element of $A(P)$ cannot be a point of accumulation for the sequence of points $\left\{U_{n}\right\}_{n=1}^{\infty}$. But as observed earlier, if $U$ is a neighborhood of the identity in $A(P)$, then $\left\{n: U_{n} \notin U\right\}$ is finite. This is a contradiction and thus $P$ is not projective.

QuEstion. A projective topological group is algebraically free on a set $S$. If $S$ is finite we know (Theorem 2) that $P$ is a free Abelian topological group (in fact is discrete). Example 1 guarantees that there are connected projective topological groups. But a connected completely regular space containing more than one point has cardinality not less than $2{ }^{N_{0}}$. The question is then: "Is there a countably infinite projective topological group that is not a free Abelian topological group?"

\section{REFERENCES}

1. R. Dudley, Continuity of homomorphisms, Duke Math. J. 28 (1961), 587-594.

2. M. Graev, Svobodnye topologičeskie gruppy, Izv. Akad. Nauk SSSR Ser. Mat. 12 (1948), 279-324; English transl., Translation No. 35, Amer. Math. Soc., Providence, R. I., 1951, 1-61; reprint, Amer. Math. Soc. Transl. (1) 8 (1962), 305-364.

3. E. Hewitt and K. A. Ross, Abstract harmonic analysis, Academic Press, New York, 1963.

4. K. Hofmann, Zerfallung topologischer Gruppen, Math. Z. 84 (1964), 16-37.

5. A. Markov, O svobodnyh topologičeskih gruppah, Izv. Akad. Nauk SSSR Ser. Mat. 9 (1945), 3-64; English transl., Translation No. 30, Amer. Math. Soc., Providence, R. I., 1950, 11-88; reprint, Amer. Math. Soc. Transl. (1) 8 (1962), 195-273.

Virginia Polytechnic Institute 